\title{
ESTRATIFICAÇÃO DA CAPACIDADE FUNCIONAL DE IDOSOS EM INSTITUIÇÃO DE LONGA PERMANÊNCIA DURANTE A PANDEMIA
}

\author{
Maria Cristina Sant'Anna da Silva' \\ ORCID: 0000-0002-9624-8152 \\ Willi Wetzel Junior" \\ ORCID: 0000-0002-6193-4064 \\ Raquel Souza Azevedo"II \\ ORCID: 0000-0002-9850-9336
}

Miria Elisabete Bairros de Camargo'v

ORCID: 0000-0003-3173-7681

Marinês Aires $\mathrm{v}$

ORCID: 0000-0002-8257-2955

Ana Karina Silva da Rocha Tanakavi

ORCID: 0000-0003-2488-3656

Letice Dalla Lana ${ }^{\text {VII }}$

ORCID: 0000-0002-9624-8152

'Enfermeira. Sociedade Brasileira de Geriatria e Gerontologia-RS. Porto Alegre, Rio Grande do Sul, Brasil.

"Enfermeiro. Quinta Urbana Hotelaria Ocupacional Assistida para Idosos. Pelotas, Rio Grande do Sul, Brasil.

"'Enfermeira. Hospital de Clínicas da Universidade Federal de Minas Gerais. Belo Horizonte, Minas Gerais, Brasil.

IVEnfermeira. Universidade Luterana do Brasil. Canoas, Rio Grande do Sul, Brasil.

vEnfermeira. Universidade Regional Integrada do Alto Uruguai e das Missões.

Frederico Westphalen, Rio Grande do Sul, Brasil.

v'Enfermeira. Universidade Federal do Rio Grande do Sul. Porto Alegre, Rio Grande do Sul, Brasil.

VIIEnfermeira. Universidade Federal do Pampa. Uruguaiana, Rio Grande do Sul, Brasil.

Autor Correspondente: Maria Cristina Sant'Anna da Silva E-mail:mcrisil@terra.com.br

\section{INTRODUÇÃO}

A longevidade da população traz importantes repercussões em diversos setores da sociedade, tornando um desafio atender as demandas assistenciais desde grupos emergentes. No Brasil, o envelhecimento acontece de modo acelerado, em cenários diversos, com grandes desigualdades sociais e culturais, com prevalência de doenças crônicas, fragmentação dos serviços de saúde, predomínio de modelo de atenção às demandas espontâneas, insuficiência de uma rede de apoio formal e mudanças na estrutura familiar ${ }^{(1-2)}$.

O envelhecer é um processo natural durante a vida e ocorre por meio de mudanças físicas, psicológicas e sociais. Com o avanço da idade, têm início alterações estruturais e funcionais, encontradas em todos os idosos, que são próprias do processo de envelhecimento normal, sendo que envelhecer sem alguma doença crônica é ocasional. Simultaneamente, ao envelhecer com a presença de doenças, aumenta a possibilidade de casos de incapacidade funcional na velhice ${ }^{(3)}$.

A incapacidade funcional torna as pessoas vulneráveis, fazendo com que necessitem de ajuda para realizar tarefas básicas ou complexas no seu dia a dia, como as atividades básicas da vida diária (ABVDs) ou as atividades instrumentais da vida diária (AIVDs). Nas ABVDs, são avaliados os comportamentos básicos e habituais de autocuidado, como a capacidade de alimentar-se, banhar-se e vestir-se. Por sua vez, as AIVDs referem-se às tarefas mais complexas e relacionadas com a autonomia e a participação social, como capacidade de realizar compras, atender ao telefone e utilizar meios de transporte ${ }^{(4)}$. Acrescenta-se as Atividades Avançadas de Vida Diária (AAVDs), as quais são mais complexas e estão relacionadas à integração social, como atividades produtivas, recreativas e de participação social(3).

$\mathrm{Na}$ atual pandemia da doença classificada como COVID-19, o agente causador de uma série de casos de pneumonias é o coronavírus, que foi nomeado como SARS-CoV-2 em $2019^{(5)}$. O vírus tem alta transmissibilidade e provoca 
síndrome respiratória aguda, de leve a muito grave, causando insuficiência, sua letalidade varia principalmente conforme a faixa etária e as condições clínicas associadas ${ }^{(5)}$.

A população idosa que reside nas Instituições de Longa Permanência para Idosos (ILPIs), de maneira geral, é considerada vulnerável por apresentar níveis variados de dependência e possuir necessidades complexas ${ }^{(6)}$. Frente a pandemia da COVID-19, torna-se ainda mais vulnerável, tendo em vista que as ILPIs são ambientes compartilhados por diversas pessoas, favorecendo a disseminação da doença.

Para proteger os residentes da COVID-19 é necessário implementar as medidas já bem estabelecidas para evitar contaminação. Além disso, mensurar o Índice de Vulnerabilidade Clínico-funcional (IVCF-20), que contempla aspectos multidimensionais da condição de saúde da pessoa idosa, possibilita a adequação do plano terapêutico singular e das rotinas institucionais, bem como a tomada de decisão sobre o uso das tecnologias de cuidado, salientando-se que quanto mais alto o valor obtido na estratificação maior o risco de vulnerabilidade ${ }^{(3)}$.

\section{OBJETIVO}

Refletir sobre a importância da estratificação da capacidade funcional das pessoas idosas institucionalizadas para o planejamento da assistência de Enfermagem perante a pandemia da covid-19.

\section{MÉTODOS}

Trata-se de estudo teórico-reflexivo sobre a utilização do Índice de Vulnerabilidade Clínico-Funcional (IVCF-20) pelo enfermeiro para a estratificação dos indivíduos idosos que residem em ILPI, visando instituir as melhores práticas de cuidado frente a pandemia da covid-19.

A análise das reflexões baseou-se sobre a utilização do IVCF-20. A estratificação clínico-funcional do IVCF20 é composta por 10 estratos, que resulta em 3 categorias: idoso robusto (estrato 1 ao 3), idoso em risco de fragilização (estrato 4 e 5) e idoso frágil (estrato 6 ao 10) - sendo que o estrato 10 corresponde ao grau máximo de fragilidade ${ }^{(3)}$.

Justifica-se o uso da Classificação Clínico-Funcional do Idoso pelo enfermeiro nas ILPIs, pois a definição de critérios de exclusão de tratamento invasivo baseada exclusivamente na faixa etária é uma medida de alocação de recursos temerária, que não deveria ser preconizada durante a pandemia da COVID-19. Além disso, acredita-se que a pessoa idosa extremamente frágil, estratificada em 9 e 10, deve ter como foco principal a proporcionalidade terapêutica, na qual deve ser priorizado o seu conforto e de seus familiares. Com base na avaliação clínica, sócio-familiar e das AVDs detalhadas no IVCF-20, o enfermeiro planeja o cuidado individual e integral à pessoa idosa em ILPI, levando em conta a identificação de fatores de risco que podem agravar-Ihe a saúde na pandemia da COVID-19.

\section{RESULTADOS}

A base do conceito de saúde das pessoas idosas é a funcionalidade global e a capacidade individual de satisfazer as necessidades biopsicossociais. A capacidade funcional é resultante da combinação entre fatores intrínsecos, como capacidade física e mental, e extrínsecos, como ambiente e contexto(3).

A prevalência da incapacidade aumenta com a idade, mas a idade sozinha não prediz incapacidade. Na perspectiva da vulnerabilidade, é possível definir a presença de declínio funcional como o principal determinante da presença de fragilidade. O termo fragilidade é comumente utilizado para representar o grau de vulnerabilidade da pessoa idosa a desfechos adversos, como declínio funcional, quedas, internação hospitalar, institucionalização e óbito. Todavia, o termo apresenta várias definições, dependendo da dimensão utilizada como referência. 
É considerada fragilidade multidimensional a redução da reserva homeostática ou da capacidade de adaptação às agressões biopsicossociais com aumento da vulnerabilidade ao declínio funcional e suas consequências ${ }^{(7)}$. Tais aspectos são indicadores importantes para que os serviços de saúde planejem ações visando prevenir ou postergar a incapacidade funcional da pessoa idosa, preservando sua autonomia, independência e qualidade de vida.

Com o processo de envelhecimento, as pessoas ficam mais susceptíveis às doenças. No cenário da pandemia da covid-19, as que são idosas estão mais expostas por apresentarem alterações decorrentes do próprio envelhecimento fisiológico e pela presença de morbidades que levam a um processo de senilidade. $\mathrm{O}$ impacto na saúde é decorrente das características individuais, mas também deve-se levar em consideração a diversidade, a pluralidade e a complexidade do processo fisiológico de envelhecer, que torna os indivíduos idosos mais vulneráveis e, por isso, grupo de risco para a pandemia da covid-19(8).

Portanto, a infecção pelo SARS-CoV-2 é um evento agudo que exige resposta adequada do organismo, diretamente relacionada à reserva homeostática ou capacidade intrínseca do indivíduo. Como a capacidade intrínseca ou vitalidade é extremamente variável entre as pessoas idosas, torna-se necessário implementar medidas clínicas imediatas e baseadas no resultado da mensuração dessa capacidade. Assim, deve-se avaliar e monitorar a capacidade funcional por ela ser um parâmetro da vitalidade da pessoa idosa.

Do ponto de vista clínico-funcional, os principais fatores de risco para a redução da capacidade funcional são: doenças crônico-degenerativas estabelecidas; comorbidades múltiplas, sarcopenia, comprometimento cognitivo leve e incapacidades funcionais. Além disso, em relação às questões sócio-familiares, residir em uma ILPI potencializa o declínio da capacidade funcional( ${ }^{(7)}$. Portanto, o risco de contaminação pelo SARS-CoV-2 torna-se extremamente elevado na população idosa frágil residente nesses ambientes, sendo necessário identificá-las.

Após o rastreamento das pessoas idosas institucionalizadas com maior risco de infecção pelo vírus SARS-CoV-2, que resulta da estratificação sistemática obtida pelo nível de capacidade funcional conforme o IVCF-20, o enfermeiro deve fazer o registro das frágeis, organizar uma planilha de monitoramento da saúde delas, prevendo fatores de risco, e instituir as ações de cuidado necessárias, sejam preventivas, terapêuticas, de reabilitação ou paliativas.

De acordo com a estratificação, idoso robusto é o que tem ausência de declínio funcional e de doenças, sendo independente para todas as AVDs, AIVDs e ABVDs. Por apresentar essas características, é pouco provável que esteja residindo em uma ILPI. Caso esteja, as ações de cuidado serão focadas na prevenção da COVID-19, que incluem medidas protetivas, como suspensão de visitas presenciais de familiares.

Por sua vez, idoso em risco de fragilização ou pré-frágil é o que possui algum grau de declínio funcional, o que afeta as principais dimensões da funcionalidade, a autonomia e a independência, tendo alta possibilidade de dependência funcional, o que pode levá-lo à institucionalização. Nesse caso, as ações de cuidado serão focadas na prevenção da COVID-19. Na presença do estrato 4, que refere-se a internação hospitalar recente, o residente deve manter as medidas preventivas, as quais incluem o isolamento por 14 dias, além de reabilitação, em especial a cardiopulmonar. E, se necessário, incluir outras necessidades de saúde como cuidados com o tecido tegumentar e musculoesquelético.

Finalmente, idoso frágil é o que tem declínio funcional estabelecido, apresentando ao menos uma incapacidade, e é incapaz de autogerenciar-se. A possibilidade de ser institucionalizado é alta; a maioria dos residentes encontra-se nesta classificação, são frágeis. As intervenções de cuidado serão prioritariamente de reabilitação ou paliativas. Em relação à essa última e frente a COVID-19, é preciso que o enfermeiro e a equipe multidisciplinar conheçam o desejo do idoso e da sua família, de forma a instituírem medidas que evitem a desproporcionalidade terapêutica, como internação hospitalar em centro de terapia intensiva e uso de ventilação mecânica.

Os cuidados paliativos devem ser planejados antecipadamente pela equipe, juntamente com a família do idoso e o próprio, se for possível. Em algumas situações, e diante da gravidade desse idoso, a família pode 
optar em não deixá-lo em unidade de cuidados intensivos, mas preferir o ambiente familiar ${ }^{(9)}$. Os cuidados paliativos são uma modalidade de atenção dirigida para cuidados ativos e totais voltados às pessoas com doenças crônicas, que visa ofertar um cuidado humanizado para promover melhor qualidade de vida e de morte, aliviando o sofrimento, a dor e demais sintomas físicos, sociais, psicológicos e espirituais.

Por conseguinte, a realização da estratificação clínico-funcional é de suma importância para a assistência de Enfermagem, pois instrumentaliza o planejamento dos cuidados para cada residente, direcionando a implementação das intervenções com base nessa estratificação. Contudo, uma intervenção de enfermagem que não está vinculada com a estratificação da capacidade clínico-funcional é a imunização dos residentes para a influenza, pois essa infecção viral não tem relação direta com o contágio da COVID-19; no entanto, se o residente apresentar sinais e sintomas gripais, a avaliação clínica poderá ficar prejudicada, daí a sua indicação.

Para melhor gestão do cuidado na ILPI, o enfermeiro deve prever consultas periódicas aos residentes, destinando atenção àqueles com estratificação 4 e 5 pelo IVCF-20, de modo a impedir que progridam para níveis mais avançados de perda funcional e tornem-se mais susceptíveis a contrair o SARS-CoV-2.

Além da participação do enfermeiro na gestão do cuidado, a equipe multidisciplinar deve contribuir com intervenções específicas. Nesse sentido, e atentando-se ao fatores de risco, como hipertensão arterial sistêmica e uso de inibidores da angiotensina, o farmacêutico em parceria com o enfermeiro devem monitorar periodicamente os sinais vitais do residente. $O$ enfermeiro ainda precisa avaliar os acamados em conjunto com o fisioterapeuta, pois a capacidade pulmonar reduzida é outro fator de risco para a infecção pelo vírus.

A perda de peso também é um fator de risco para a fragilidade e pode prejudicar a imunidade do residente. O nutricionista deve estar alerta sobre a precisão e periodicidade das avaliações nutricionais, a ingestão calórica e a disponibilização de alimentos que o residente aprecia. Mas, durante a pandemia, esses profissionais podem ter reduzido as idas à instituição pela necessidade de distanciamento social. Nesse caso, o enfermeiro deve sinalizar os técnicos de enfermagem e cuidadores quanto à importância da ingestão alimentar adequada dos residentes e à comunicação de possíveis recusas.

Assim, frente a complexidade do cuidados para as pessoas idosas institucionalizadas, em especial durante a pandemia da COVID-19, é recomendável que o enfermeiro tenha qualificação em gerontologia. Nesse sentido, apresentar condições técnicas permite que ele efetue o cuidado adequado, levando em consideração a classificação do perfil clínico-funcional dos residentes, o que aumenta a possibilidade de implantar com assertividade as melhores práticas de cuidado. Cabe ressaltar que a avaliação do residente deve ocorrer na sua admissão e sempre que ele tiver alguma intercorrência, como mudança de comportamento ou internação hospitalar ${ }^{(10)}$.

Diante do processo de envelhecimento humano, com peculiaridades próprias, aliado à alta incidência das doenças crônicas e suas repercussões, evidencia-se a necessidade de atenção multidisciplinar às pessoas idosas institucionalizados. Quanto à Enfermagem, há de pensar-se que seja valorizado o conhecimento adquirido pelos profissionais da área no transcurso da pandemia e reconhecida a necessidade deles serem qualificados para exercerem o cuidado integral aos residentes.

\section{Limitações}

Como limitações do estudo, observa-se que poucas pesquisas referentes à pandemia utilizam o IVCF-20 no cenário das ILPIs, sendo o referido instrumento mais utilizado na Atenção Primária à Saúde, pela equipe de Saúde da Família.

\section{Contribuições para enfermagem}

O presente estudo salienta a importância e necessidade de o enfermeiro realizar a estratificação e o monitoramento da capacidade funcional das pessoas idosas institucionalizadas, de modo a possibilitar a agilidade 
e a assertividade na escolha do plano de cuidados individualizado, promovendo qualidade de vida e redução de possíveis danos frente a pandemia de covid-19.

Considerando que pessoas idosas em condição de institucionalização apresentam maior dependência para as atividades básicas de vida diária, além de comprometimento da autonomia e da cognição, a avaliação clínico-funcional contribui para a tomada de decisão de instituir-lhes cuidados paliativos - sempre em consonância com os membros da equipe e os respectivos familiares -, quando for o caso. O Enfermeiro pode ser o profissional pioneiro da equipe multidisciplinar a aventar a possibilidade de implantar esse tipo de cuidado qualificado.

\section{CONSIDERAÇÕES FINAIS}

Este estudo de reflexão traz subsídios que sustentam a implementação do IVCF-20 para determinar o perfil clínico-funcional de pessoas idosas residentes em ILP, uma vez que o resultado da estratificação é importante e necessário para a elaboração do plano de cuidados frente a pandemia da covid-19, auxiliando na tomada de decisão sobre as melhores práticas a serem instituídas.

De maneira geral, os residentes de instituições de longa permanência são pessoas idosas portadoras de doenças crônicas não transmissíveis e dependentes para as atividades de vida diária, fatores que propiciam maior vulnerabilidade à infecção pelo coronavírus. Medidas de prevenção ao vírus devem ser implantadas com a maior brevidade para todos os residentes; e para aqueles em risco de fragilização, reabilitação precoce. Mas, para os residentes frágeis, cuidados paliativos poderão ser indicados durante a pandemia. Não obstante, há muito a ser estudado e concretizado na assistência de Enfermagem à população idosa institucionalizada, sendo a qualificação profissional da categoria um quesito que precisa ser estimulado e recomendado.

\section{AGRADECIMENTO}

Ao Departamento Científico de Enfermagem Gerontológica da ABEn Nacional.

\section{REFERÊNCIAS}

1. Lima-Costa MF, Peixoto SV, Malta DC, Szwarcwald CL, Mambrini JVM. Informal and paid care for Brazilian older adults (National Health Survey, 2013). Rev Saúde Pública. 2017;51(Supl 1):6s. doi: https://doi.org/10.1590/ s1518-8787.2017051000013

2. Veras RP, Oliveira M. Aging in Brazil: the building of a healthcare model. Ciênc Saúde Coletiva. 2018;23(6):1929-36. doi: https://doi.org/10.1590/1413-81232018236.04722018

3. Moraes EN, Moraes FL, Matos MAB, Lopes PRR, Chomatas ERV, Machado LC et.al. Nota Técnica para Organização da Rede de Atenção à Saúde com Foco na Atenção Primária à Saúde e na Atenção Ambulatorial Especializada - Saúde da Pessoa Idosa. São Paulo: Hospital Israelita Albert Einstein: Ministério da Saúde, 2019. Disponível em: https://atencaobasica.saude. rs.gov.br/upload/arquivos/202001/03091212-nt-saude-do-idoso-planificasus.pdf

4. Farias-Antúnez S, Lima NP, Bierhals IO, Gomes AP, Vieira LS, Tomasi E. Incapacidade funcional para atividades básicas e instrumentais da vida diária: um estudo de base populacional com idosos de Pelotas, Rio Grande do Sul, 2014. Epidemiol Serv Saúde. 2018:27(2): e2017290. doi: https://doi.org/10.5123/s1679-49742018000200005

5. World Health Organization (WHO). Coronavirus disease 2019 (COVID-19) - Situation Report 51. Geneve: WHO [Internet]. 2020 [cited 2020 May 20]. Disponível em: https://www.who.int/docs/default-source/coronaviruse/situationreports/20200311-sitrep-51-covid-19.pdf?sfvrsn=1ba62e57_10

6. Agência Nacional de Vigilância Sanitária (ANVISA). Nota técnica n. 05/2020 GVIMS/GGTES. Orientações para a Prevenção e Controle de Infecções pelo novo coronavírus (SARS-Cov-2) em Instituições de Longa Permanência para Idosos (ILPI). Brasília, 24 de março de 2020. [Internet]. 2020. [acesso em 06 jun 2020]. Disponível em: http://portal.anvisa.gov.br/ documents/33852/271858/ NOTA+T\%C3\%89CNICA+N\%C2\%BA+05-2020+GVIMS-GGTES-ANVISA+-+ORIENTA\%C3\%8 
7. Moraes EN, Azevedo RS, Moraes FL. Saúde e envelhecimento. In: Moraes EN, Azevedo RS, organizadores. Fundamentos do cuidado ao idoso frágil. Belo Horizonte: Folium, 2016. p. 1-25.

8. Hammerschmidt KS de A, Santana RF. Saúde do idoso em tempos de pandemia Covid-19. Cogitare enferm. 2020; 25:e72849. Disponível em: http:// dx.doi.org/10.5380/ce.v25i0.72849

9. Roland K, Minder Markus M. COVID-19 pandemic: palliative care for elderly and frail patients at home and in residential and nursing homes. Swiss Med Wkly. 2020:150:w20235. doi: https://doi.org/10.4414/smw.2020.20235

10. Delvalle R, Santana RF. Avaliação inicial e sistemática de residentes. In: Silva MCS, Caberlon IC, organizadoras. Instituições de longa permanência para idosos: gerenciamento e assistência. Porto Alegre: Moriá, 2020.p 19-31. 